\title{
SYNTHESIS OF FLAVONES FROM 2-HYDROXY ACETOPHENONE AND AROMATIC ALDEHYDE DERIVATIVES BY CONVENTIONAL METHODS AND GREEN CHEMISTRY APPROACH
}

\author{
SAMIR PATEL*, UMANG SHAH \\ Department of Pharmaceutical Chemistry, Ramanbhai Patel College of Pharmacy, Charotar University of Science and Technology, Changa, \\ Gujarat, India. Email: samirpatel.ph@charusat.ac.in
}

Received: 28 October 2016, Revised and Accepted: 12 November 2016

\section{ABSTRACT}

Objective: Flavones occupy a special place in the realm of natural and synthetic organic chemistry owing to their diversified biological activities. In this study, a series of chalcone derivatives were synthesized and after cyclization of chalcone to synthesized various substituted flavone derivatives (2A-2L).

Methods: The reaction of 2-hydroxy acetophenone with substituted aromatic aldehydes produced chalcone by trituration (NaOH) and conventional methods $(\mathrm{KOH} / \mathrm{EtOH})$, which upon further cyclization with dimethyl sulfoxide $/ \mathrm{I}_{2}$ resulted to form flavone derivatives.

Results: The purity of compounds was ascertained by melting point and thin-layer chromatography. The synthesized compounds have been characterized by mass, infrared, and ${ }^{1} \mathrm{H}$ nuclear magnetic resonance spectral analysis.

Conclusion: Based on spectral data, it was proved that all synthesized chalcones and flavones derivatives meet the standard values of various spectral techniques and further it will be evaluated for pharmacological activities.

Keywords: Chalcone, Flavone, Trituration, Conventional, Claisen-Schmidt condensation.

(C) 2017 The Authors. Published by Innovare Academic Sciences Pvt Ltd. This is an open access article under the CC BY license (http://creativecommons. org/licenses/by/4. 0/) DOI: http://dx.doi.org/10.22159/ajpcr.2017.v10i2.15928

\section{INTRODUCTION}

Flavonoids are a group of more than 4000 polyphenolic compounds that occur naturally in foods of plant origin. These compounds possess a common phenylbenzopyrone structure (C6-C3-C6), and they are categorized according to the saturation level and opening of the central pyran ring, mainly into flavones, flavanols, isoflavones, flavonols, flavanones, and flavanonols [1,2]. Flavones occupy a special place in the realm of natural and synthetic organic chemistry owing to their useful biological activities such as antioxidant [3-7], anxiolytic [8], anticancer [9-11], analgesic and anti-inflammatory [12-14], antimicrobial [15], antiulcer, and thrombosis [16].

Chalcone is a starting material for the synthesis of flavones and chalcones can be synthesized by many methods. In general, chalcones were prepared by Claisen-Schmidt condensation of electrophilic substituted benzaldehyde with substituted acetophenone as nucleophile in the presence of bases such as $\mathrm{NaOH}, \mathrm{KOH}, \mathrm{Ba}(\mathrm{OH})_{2}, \mathrm{LiOH}, \mathrm{NaH}$, hydrotalcites, Zeolites, $\mathrm{Na}_{2} \mathrm{CO}_{3}, \mathrm{~K}_{2} \mathrm{CO}_{3}$, magnesium t-butoxide, alumina, $\mathrm{MgO}, \mathrm{KF} /$ natural phosphate, calcined $\mathrm{NaNO}_{3}$-natural phosphates, and piperidine. Chalcones are also prepared by ultrasonic vibration and microwave irradiation techniques [17-19].

Green chemistry is the need of the day and hence it was planned to synthesize some chalcones in an eco-friendly way without using solvents. Thus, the synthesis involves the solvent-free solid state trituration methods involved Claisen-Schmidt reaction between acetophenone derivatives and substituted benzaldehydes in the presence of $\mathrm{NaOH}$. The remaining chalcone was planned to synthesize by taking $\mathrm{KOH}$ as a base. Using these chalcone derivatives, it was contemplated to synthesis of some flavone derivatives from the corresponding chalcone by using dimethyl sulfoxide (DMSO)/ $\mathrm{I}_{2}[14]$.

\section{METHODS}

All the chemicals were obtained from commercial sources and used without further purification. Melting point was measured in digital melting point apparatus (Veego, VMP-DS) model. Infrared (IR) and mass spectra of synthesized compounds were taken by using IR spectrometer (NICOLET 6700, Thermo Scientific) and mass spectrometer (Advion Compact Mass Spectrometer) by ESI Techniques, respectively. ${ }^{1} \mathrm{H}$ nuclear magnetic resonance (NMR) spectral was recorded at room temperature on a $400 \mathrm{MHz}$ liquid state NMR spectrometer in DMSO-d6 (Brüker Biospin, Switzerland) using tetramethylsilane as internal standard. The reactions were monitored by thin-layer chromatography (TLC) using precoated plates (Merck). All solvents used in thin layer chromatography were distilled before use.

\section{General procedure for the synthesis of substituted chalcone} (1A-1L, Scheme 1)

\section{Method $1(1 A-1 E)$}

About $24 \mathrm{mmol}$ of aryl aldehyde (1.2 equivalent) were taken in mortal pestle triturated with $\mathrm{NaOH}$ powder added in portion wise with continuous trituration. $20 \mathrm{mmol} 2$-hydroxy acetophenone (1 equivalent) was added with continuous trituration. A solid yellow mass was formed with continuous trituration. The reaction was monitored by TLC. The formed yellow solid was immediately washed with hot methanol to get crude chalcone.

\section{Method 2 (1F-1S)}

2-Hydroxy acetophenone (1 equivalent) and benzaldehyde derivatives (1.2 equivalent) were dissolved in $\mathrm{EtOH}$ and $\mathrm{KOH}$ pallet (3 equivalent) was added. The reaction mixture was stirred at RT for 6-12 hrs until reaction completion was indicated by TLC. The reaction was worked up the mixture was poured onto crushed ice and acidified with dilute $\mathrm{HCl}(\mathrm{pH}$ 5). The solid was recrystallized from dilute ethanol to get crystalline chalcone.

General procedure for the synthesis of all substituted flavones (2A-2L)

Synthesized 2-hydroxy arylchalcone ( $5 \mathrm{mmol}$ ) was taken in radial basis function, and $6 \mathrm{ml}$ of DMSO was added in it. Then, catalytic amount 
of $\mathrm{I}_{2}$ was added to the reaction mixture and heat the content on oil bath at $110^{\circ} \mathrm{C}$ for $2-6 \mathrm{hrs}$. The reaction completion was indicated by TLC. The reaction was worked up the mixture was poured onto crushed ice and excess $\mathrm{I}_{2}$ was removed by slow addition of sodium thiosulfate solution. The precipitated product was filtered by suction and solid was recrystallized from dilute ethanol to get crystalline flavone (Scheme 1).

\section{RESULTS AND DISCUSSION}

Synthesis of chalcone was achieved by claisen-schmidt condensation of 2-hydroxy acetophenone and various aromatic aldehyde derivatives by solvent free trituration $(1 \mathrm{~A}-1 \mathrm{~F})$ and conventional (1G-1L) method. From synthesized chalcone derivatives various flavone derivatives were synthesized. The synthesized chalcone was confirmed by various physicochemical means, TLC and IR spectrometer. The IR absorption spectrum band at 3600-3400 $\mathrm{cm}^{-1}$ indicated that the presence of hydroxyl groups and $1600-1660 \mathrm{~cm}^{-1}$ indicated the presence of $\alpha, \beta$ unsaturated carbonyl $(>\mathrm{C}=0$ ) carbon in synthesized chalcone (Fig. 1 and Table 1).

Cyclization of chalcone into corresponding flavones was carried out using DMSO $/ \mathrm{I}_{2}$ as catalyst. The formation of flavones has been supported by TLC, physicochemical means and various spectral techniques (IR, mass and ${ }^{1} \mathrm{H}$ NMR). In flavones, the IR absorption spectrum band at $3020-3070 \mathrm{~cm}^{-1}$ indicated that the presence of aromatic(-C-H) stretching and $1600-1660 \mathrm{~cm}^{-1}$ indicated the presence of $\alpha, \beta$ unsaturated carbonyl ( $>C=0$ ) carbon in synthesized flavones and absence of $-\mathrm{OH}$ group band confirmed the oxidation of chalcone into flavones. ${ }^{1} \mathrm{H}$ NMR and mass spectral data also supported the formation of flavones derivatives as shown in spectral data section.

\section{Spectral data of flavone derivatives}

\section{2-(2,3-dimethoxyphenyl)-4H-chromen-4-one (2A)}

Molecular formula: $\mathrm{C}_{17} \mathrm{H}_{14} \mathrm{O}_{4}$, yield: $78 \%$, MP: $142-145^{\circ} \mathrm{C}$, IR $(\mathrm{KBr}) \mathrm{cm}^{-1}: 3062$ (-CH aromatic str), 1653 (>C=0 str), 1258, 1152
(R-0-Arstr), ${ }^{1} \mathrm{H}$ NMR (DMSO-d6), $400 \mathrm{MHz}, \delta$ (ppm): 3.92 (s, 6H, - $\mathrm{OCH}_{3}$ ), $6.69(\mathrm{~s}, 1 \mathrm{H}$, pyrone ring), $7.60(\mathrm{t}, 2 \mathrm{H}$, phenyl ring $\mathrm{A}), 7.35(\mathrm{~d}, 2 \mathrm{H}$, phenyl ring A), 8.02 (d, 1H, Ar-CH, ring B), 6.91 (d, 1H, Ar-CH, ring B), 7.19 (s, $1 \mathrm{H}, \mathrm{Ar}-\mathrm{CH}$, ring B); Mass: $283.3(\mathrm{M}+1)$.

\section{2-(3-hydroxyphenyl)-4H-chromen-4-one (2B)}

Molecular formula: $\mathrm{C}_{15} \mathrm{H}_{10} \mathrm{O}_{3}$, yield: $59 \%$, MP: $139-142^{\circ} \mathrm{C}$, IR (KBr) $\mathrm{cm}^{-1}: 3089$ (-CH aromatic str), 3461 (-OH str), 1653 (>C=0 str); ${ }^{1} \mathrm{H}$ NMR (DMSO-d6), $400 \mathrm{MHz}, \delta$ (ppm): 8.48 (s, 1H, -OH), 6.79 (s, 1H, pyrone ring), 7-7.90 (m, 4H, Ar- $\mathrm{CH}$, ring A), 7.37-7.90 (m, 4H, Ar- $\mathrm{CH}$, ring B); mass: $239.2(\mathrm{M}+1)$

\section{2-(4-methoxyphenyl)-4H-chromen-4-one (2C)}

Molecular formula: $\mathrm{C}_{16} \mathrm{H}_{12} \mathrm{O}_{3}$, yield: $75 \%$, MP: $145-148^{\circ} \mathrm{C}$, IR (KBr) $\mathrm{cm}^{-1}: 3059$ (-CH Aromatic str), 1628 (>C=0 str), 1247, 1134 (R-O-Arstr); ${ }^{1} \mathrm{H}$ NMR (DMSO-d6), $400 \mathrm{MHz}, \delta$ (ppm): 3.83 (s, 3H, - $\mathrm{OCH}_{3}$ ) 6.69 (s, 1H, pyrone ring), $7.34(\mathrm{t}, 1 \mathrm{H}, \mathrm{Ar}-\mathrm{CH}$, ring $\mathrm{A}), 7.60(\mathrm{t}, 1 \mathrm{H}, \mathrm{Ar}-\mathrm{CH}$, ring $\mathrm{A}), 8.17$ (d, 1H, Ar-CH, ring A), 7.50 (d, 1H, Ar-CH, ring A), 6.95 (dd, 2H, Ar-CH, ring B), 7.81 (dd, 2H, Ar-CH, ring B); Mass: $253.3(\mathrm{M}+1)$.

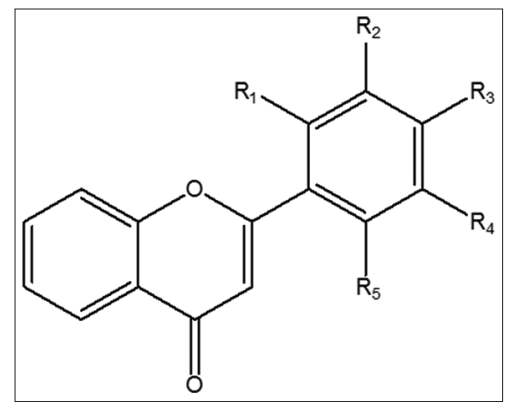

Fig. 1: General structure of chalcone

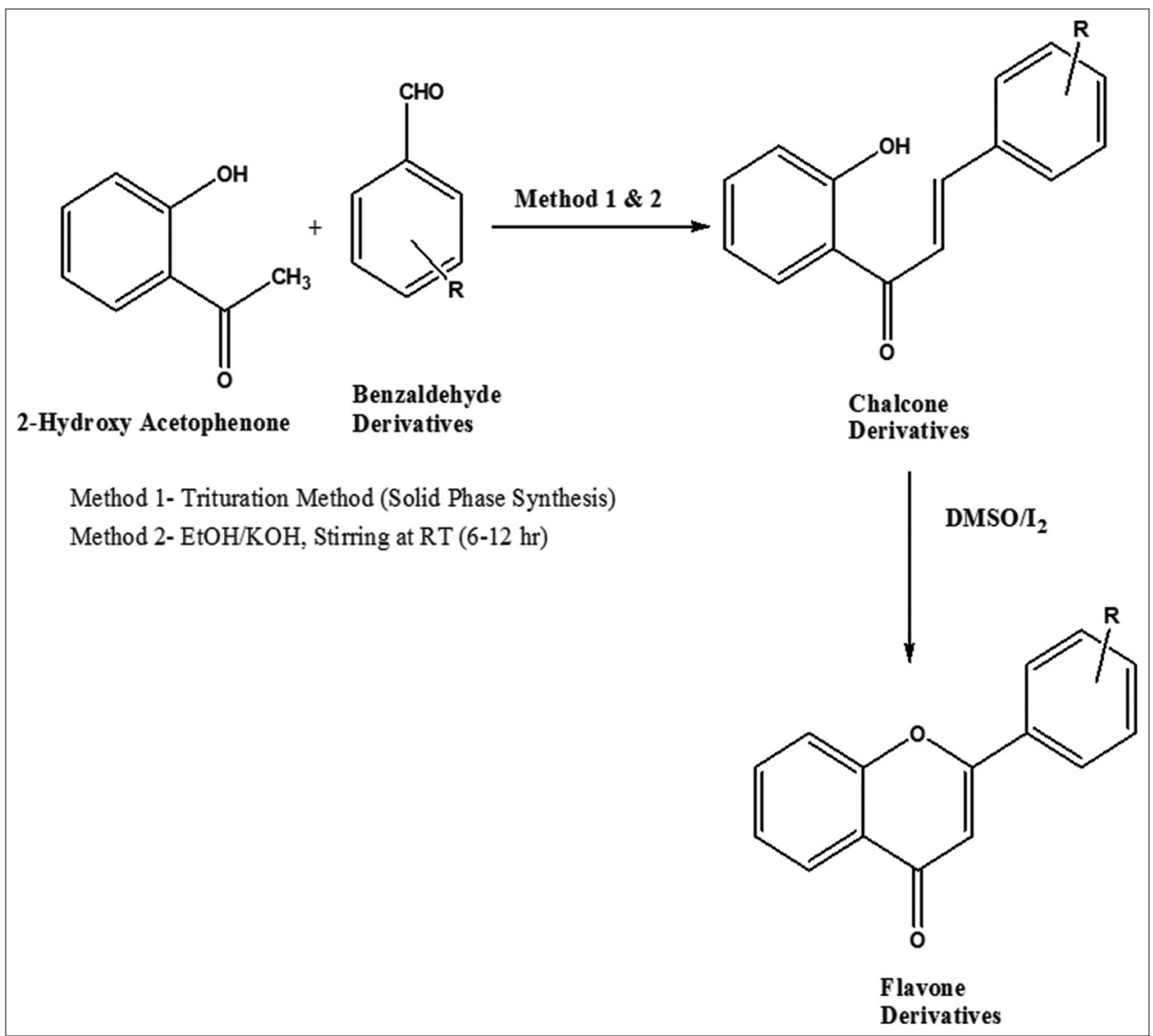

Scheme 1: Synthesis of chalcones by trituration (1A-1F) and conventional method (1G-1L) and flavone (2A-2L) 
Table 1: Physicochemical and IR spectral data of chalcone derivatives

\begin{tabular}{|c|c|c|c|c|c|c|c|c|}
\hline $\begin{array}{l}\text { Compound } \\
\text { ID }\end{array}$ & $\mathbf{R}_{1}$ & $\mathbf{R}_{2}$ & $\mathbf{R}_{3}$ & $\mathbf{R}_{4}$ & $\mathbf{R}_{5}$ & $\begin{array}{l}\text { Melting } \\
\text { point }\left({ }^{\circ} \mathrm{C}\right)\end{array}$ & Yield (\%) & $\operatorname{IR}\left(\mathrm{cm}^{-1}\right)$ \\
\hline $1 \mathrm{~A}$ & $-\mathrm{H}$ & $-\mathrm{OCH}_{3}$ & $-\mathrm{OCH}_{3}$ & $-\mathrm{H}$ & $-\mathrm{H}$ & $92-94$ & 86 & $\begin{array}{l}3001 \text { (Aromatic -C-H) str., 3600-3200 (O-H) str., } \\
1695 \text { (C=O) str., 1513, } 1465 \text { (C=C) str., 1266, } 1152 \text { (R-O-Ar) } \\
\text { str. }\end{array}$ \\
\hline $1 \mathrm{~B}$ & $-\mathrm{H}$ & $-\mathrm{OH}$ & $-\mathrm{H}$ & $-\mathrm{H}$ & $-\mathrm{H}$ & $85-88$ & 78 & $\begin{array}{l}3030 \text { (Aromatic }-\mathrm{C}-\mathrm{H}) \text { str., } 3600-3200(\mathrm{O}-\mathrm{H}) \text { str., } \\
1640(\mathrm{C}=0) \text { str., } 1488(\mathrm{C}=\mathrm{C}) \text { str. }\end{array}$ \\
\hline $1 \mathrm{C}$ & $-\mathrm{H}$ & $-\mathrm{H}$ & $-\mathrm{OCH}_{3}$ & $-\mathrm{H}$ & $-\mathrm{H}$ & $78-80$ & 83 & $\begin{array}{l}3065 \text { (Aromatic-C-H) str., } 3600-3200 \text { (O-H) str., } \\
1637 \text { (C=O) str., 1581, } 1488 \text { (C=C) str., 1261, } 1152 \text { (R-O-Ar) } \\
\text { str. }\end{array}$ \\
\hline 1D & $-\mathrm{H}$ & $-\mathrm{H}$ & $-\mathrm{Cl}$ & $-\mathrm{H}$ & $-\mathrm{H}$ & $82-84$ & 68 & $\begin{array}{l}3055 \text { (Aromatic -C-H) str., 3600-3200 (O-H) str., } \\
1635 \text { (C=0) str., 1558, } 1488 \text { (C=C) str., } 746 \text { (Ar-Cl) str. }\end{array}$ \\
\hline $1 \mathrm{E}$ & $-\mathrm{H}$ & $-\mathrm{H}$ & $-F$ & $-\mathrm{H}$ & $-\mathrm{H}$ & $68-70$ & 85 & $\begin{array}{l}3055 \text { (Aromatic -C-H) str., 3600-3200 (O-H) str., } \\
1589 \text { (C=0) str., 1513, } 1494 \text { (C=C) str. }\end{array}$ \\
\hline $1 \mathrm{~F}$ & $-\mathrm{H}$ & $-\mathrm{Cl}$ & $-\mathrm{H}$ & $-\mathrm{H}$ & $-\mathrm{H}$ & $80-83$ & 75 & $\begin{array}{l}3056 \text { (Aromatic -C-H) str., 3600-3200 (O-H) str., } \\
1647 \text { (C=0) str., 1583, } 1491 \text { (C=C) str., } 755 \text { (Ar-Cl) str. }\end{array}$ \\
\hline $1 \mathrm{G}$ & $-\mathrm{H}$ & $-\mathrm{OCH}_{3}$ & $-\mathrm{H}$ & $-\mathrm{H}$ & $-\mathrm{H}$ & $65-68$ & 88 & $\begin{array}{l}3010 \text { (Aromatic -C-H) str., 3600-3200 (O-H) str., } \\
1640 \text { (C=O) str., 1576, } 1486 \text { (C=C) str., 1271, } 1157 \text { (R-O-Ar) } \\
\text { str. }\end{array}$ \\
\hline $1 \mathrm{H}$ & $-\mathrm{OCH}_{3}$ & $-\mathrm{H}$ & $-\mathrm{OCH}_{3}$ & $-\mathrm{H}$ & $-\mathrm{OCH}_{3}$ & $108-110$ & 78 & $\begin{array}{l}3035 \text { (Aromatic -C-H ) str., } 3600-3200 \text { (O-H) str., } \\
1624 \text { (C=O) str., 1576, } 1488 \text { (C=C) str., } 1268,1156 \text { (R-O-Ar) } \\
\text { str. }\end{array}$ \\
\hline $1 \mathrm{I}$ & $-\mathrm{OCH}_{3}$ & $-\mathrm{OCH}_{3}$ & $-\mathrm{H}$ & $-\mathrm{H}$ & $-\mathrm{H}$ & $115-117$ & 86 & $\begin{array}{l}3065 \text { (Aromatic -C-H) str., 3600-3200 (O-H) str., } \\
1640 \text { (C=O) str., 1577, } 1487 \text { (C=C) str., 1272, } 1159 \text { (R-O-Ar) } \\
\text { str. }\end{array}$ \\
\hline 1J & $-\mathrm{OCH}_{3}$ & $-\mathrm{H}$ & $-\mathrm{OCH}_{3}$ & $-\mathrm{H}$ & $-\mathrm{H}$ & $106-108$ & 75 & $\begin{array}{l}3001 \text { (Aromatic -C-H ) str., } 3600-3200 \text { (O-H) str., } \\
1636 \text { (C=0) str., 1560, } 1489 \text { (C=C) str, 1282, } 1158 \text { (R-O-Ar) } \\
\text { str. }\end{array}$ \\
\hline $1 \mathrm{~K}$ & $-\mathrm{H}$ & $-\mathrm{H}$ & $-\mathrm{H}$ & $-\mathrm{H}$ & $-\mathrm{H}$ & $78-80$ & 86 & $\begin{array}{l}3045 \text { (Aromatic -C-H) str., 3600-3200 (O-H) str., } \\
1640 \text { (C=0) str., 1574, } 1485 \text { (C=C) str., }\end{array}$ \\
\hline $1 \mathrm{~L}$ & $-\mathrm{H}$ & $-\mathrm{Br}$ & $-\mathrm{H}$ & $-\mathrm{H}$ & $-\mathrm{H}$ & $108-110$ & 75 & $\begin{array}{l}3058 \text { (Aromatic -C-H) str., 3600-3200 (O-H) str., } 1641(\mathrm{C}=0) \\
\text { str., } 1575,1485(\mathrm{C}=\mathrm{C}) \text { str. }\end{array}$ \\
\hline
\end{tabular}

IR: Infrared

\section{2-(4-chlorophenyl)-4H-chromen-4-one (2D)}

Molecular formula: $\mathrm{C}_{15} \mathrm{H}_{9} \mathrm{ClO}_{2}$, yield: $65 \%$, MP: $154-157^{\circ} \mathrm{C}$, IR $(\mathrm{KBr})$ $\mathrm{cm}^{-1}$ : 3066(-CH Aromatic str), 1621 (>C=0 str), 771 (Ar-Clstr); ${ }^{1} \mathrm{H}$ NMR (DMSO-d6), $400 \mathrm{MHz}, \delta$ (ppm): 6.98 (s, 1H, pyrone ring), 7.13-8.31 (m, 4H, Ar-CH, ring A), 7.57 (dd, 2H, Ar-CH, ring B), 7.70 (dd, 2H, Ar-CH, ring B); mass: $257.2(\mathrm{M}+1)$.

\section{2-(4-fluorophenyl)-4H-chromen-4-one (2E)}

Molecular formula: $\mathrm{C}_{15} \mathrm{H}_{9} \mathrm{FO}_{2}$, yield: $68 \%$, MP: $152-154^{\circ} \mathrm{C}$, IR $(\mathrm{KBr})$ $\mathrm{cm}^{-1}: 3045$ (-CH Aromatic str), 1601 (>C=0 str); ${ }^{1} \mathrm{H}$ NMR (DMSO-d6), $400 \mathrm{MHz}, \delta$ (ppm): $6.73(\mathrm{~s}, 1 \mathrm{H}$, pyrone ring), $7.34(\mathrm{t}, 1 \mathrm{H}, \mathrm{Ar}-\mathrm{CH}$, ring $\mathrm{A}$ ), 7.67 (t, $1 \mathrm{H}, \mathrm{Ar}-\mathrm{CH}$, ring A), 7.51 (d, $1 \mathrm{H}, \mathrm{Ar}-\mathrm{CH}$, ring A), 8.17 (d, $1 \mathrm{H}, \mathrm{Ar}-$ $\mathrm{CH}$, ring $\mathrm{A}$ ), 7.45 (dd, 2H, Ar- $\mathrm{CH}$, ring B), 7.81 (dd, 2H, Ar-CH, ring B); mass: $241.2(\mathrm{M}+1)$.

\section{2-(3-chlorophenyl)-4H-chromen-4-one (2F)}

Molecular formula: $\mathrm{C}_{15} \mathrm{H}_{9} \mathrm{ClO}_{2}$, yield: $85 \%$, MP: $90-94^{\circ} \mathrm{C}$, IR (KBr) $\mathrm{cm}^{-1}: 3030$ (-CH Aromatic str), 1644 (>C=0 str), 693 (Ar-Clstr); ${ }^{1} \mathrm{H}$ NMR (DMSO-d6), $400 \mathrm{MHz}, \delta$ (ppm): $7.16(\mathrm{~s}, 1 \mathrm{H}$, pyrone ring), $7.72(\mathrm{t}, 1 \mathrm{H}, \mathrm{Ar}-$ $\mathrm{CH}$, ring $\mathrm{A}$ ), 7.67 (t, $1 \mathrm{H}, \mathrm{Ar}-\mathrm{CH}$, ring $\mathrm{A}), 8.09$ (d, $1 \mathrm{H}, \mathrm{Ar}-\mathrm{CH}$, ring $\mathrm{A}), 8.12$ (d, $1 \mathrm{H}, \mathrm{Ar}-\mathrm{CH}$, ring A), $8.22(\mathrm{~s}, 1 \mathrm{H}, \mathrm{Ar}-\mathrm{CH}$, ring $\mathrm{B}$ ), 7.90 (d, $1 \mathrm{H}, \mathrm{Ar}-\mathrm{CH}$, ring B), $8.22(\mathrm{~s}, 1 \mathrm{H}, \mathrm{Ar}-\mathrm{CH}$, ring B), $7.98(\mathrm{t}, 1 \mathrm{H}, \mathrm{Ar}-\mathrm{CH}$, ring B), $7.95(\mathrm{~d}, 1 \mathrm{H}$, Ar- $\mathrm{CH}$, ring $\mathrm{B})$; mass: $257.3\left(\mathrm{M}^{+}\right)$.

\section{2-(3-methoxyphenyl)-4H-chromen-4-one (2G)}

Molecular formula: $\mathrm{C}_{16} \mathrm{H}_{12} \mathrm{O}_{3}$, yield: $79 \%$, MP: $64-67^{\circ} \mathrm{C}$, IR $(\mathrm{KBr})$ $\mathrm{cm}^{-1}: 3045$ (-CH Aromatic str), 1629 (>C=0 str), 1292, 1099 (R-0-Arstr); ${ }^{1} \mathrm{H}$ NMR (DMSO-d6), $400 \mathrm{MHz}, \delta$ (ppm): 3.95 (s, 3H, - $\mathrm{OCH}_{3}$ ) 6.62 (s, $1 \mathrm{H}$, pyrone ring), $7.84(\mathrm{t}, 1 \mathrm{H}, \mathrm{Ar}-\mathrm{CH}$, ring $\mathrm{A}), 7.51(\mathrm{t}, 1 \mathrm{H}, \mathrm{Ar}-\mathrm{CH}$, ring $\mathrm{A}), 7.69$ (d, 1H, Ar-CH, ring A), 8.05 (d, 1H, Ar-CH, ring A), 6.26, 6.42 (m, 2H, Ar$\mathrm{CH}$, ring $\mathrm{B}), 7.31,7.33(\mathrm{~m}, 2 \mathrm{H}, \mathrm{Ar}-\mathrm{CH}$, ring $\mathrm{B})$; Mass: $252.2\left(\mathrm{M}^{+}\right)$.

\section{2-(2,4,6-trimethoxyphenyl)-4H-chromen-4-one (2H)}

Molecular formula: $\mathrm{C}_{18} \mathrm{H}_{16} \mathrm{O}_{5}$, Yield: $66 \%$, MP: $90-94^{\circ} \mathrm{C}$, IR (KBr) $\mathrm{cm}^{-1}: 3030$ (-CH Aromatic str), 1645 ( $>\mathrm{C}=0 \mathrm{str}$ ), 1279, 1157 (R-O-Arstr); ${ }^{1} \mathrm{H}$ NMR (DMSO-d6), $400 \mathrm{MHz}, \delta$ (ppm): 3.95 (s, 9H, - $\mathrm{OCH}_{3}$ ) 6.62 (s, 1H, pyrone ring), $7.80(\mathrm{t}, 1 \mathrm{H}, \mathrm{Ar}-\mathrm{CH}$, ring $\mathrm{A}), 7.51(\mathrm{t}, 1 \mathrm{H}, \mathrm{Ar}-\mathrm{CH}$, ring $\mathrm{A}), 7.69$ $(\mathrm{m}, 1 \mathrm{H}, \mathrm{Ar}-\mathrm{CH}$, ring $\mathrm{A}), 8.05(\mathrm{~m}, 1 \mathrm{H}, \mathrm{Ar}-\mathrm{CH}$, ring $\mathrm{A}), 6.26,6.42(\mathrm{~m}, 2 \mathrm{H}$, Ar- $\mathrm{CH}$, ring B), mass: $283.3(\mathrm{M}+1)$.

\section{2-(2,3-dimethoxyphenyl)-4H-chromen-4-one (2I)}

Molecular formula: $\mathrm{C}_{17} \mathrm{H}_{14} \mathrm{O}_{4}$, Yield: $84 \%$, MP: $102-105^{\circ} \mathrm{C}$, IR (KBr) $\mathrm{cm}^{-1}$ : 3038 (-CH Aromatic str), 1641 (>C=0 str), 1256, 1142 (R-OArstr); ${ }^{1} \mathrm{H}$ NMR (DMSO-d6), $400 \mathrm{MHz}, \delta$ (ppm): 3.00, 3.17 (s, 6H, $-\mathrm{OCH}_{3}$ ) $6.78(\mathrm{~s}, 1 \mathrm{H}$, pyrone ring), $7.84(\mathrm{t}, 1 \mathrm{H}, \mathrm{Ar}-\mathrm{CH}$, ring A), $7.52(\mathrm{t}, 1 \mathrm{H}, \mathrm{Ar}-\mathrm{CH}$, ring $\mathrm{A}), 7.38(\mathrm{~d}, 1 \mathrm{H}, \mathrm{Ar}-\mathrm{CH}$, ring $\mathrm{A}), 8.08(\mathrm{~d}, 1 \mathrm{H}, \mathrm{Ar}-\mathrm{CH}$, ring $\mathrm{A}), 7.31,7.27$ (m, 2H, Ar-CH, ring B), 7.75 (d, 1H, Ar-CH, ring B) mass: $283.3(\mathrm{M}+1)$.

\section{2-(2,4-dimethoxyphenyl)-4H-chromen-4-one (2J)}

Molecular formula: $\mathrm{C}_{17} \mathrm{H}_{14} \mathrm{O}_{4^{\prime}}$ yield: $76 \%$, MP: 80-84 ${ }^{\circ} \mathrm{C}$, IR (KBr) cm ${ }^{-1}: 3056$ (-CH Aromatic str), 1629 ( $>\mathrm{C}=0$ str), 1280, 1152 (R-0-Arstr); ${ }^{1} \mathrm{H}$ NMR (DMSO-d6), $400 \mathrm{MHz}, \delta$ (ppm): 3.97 (s, 6H, - $\mathrm{OCH}_{3}$ ) 6.93 (s, $1 \mathrm{H}$, pyrone ring), $7.80(\mathrm{t}, 1 \mathrm{H}, \mathrm{Ar}-\mathrm{CH}$, ring $\mathrm{A}), 7.48(\mathrm{t}, 1 \mathrm{H}, \mathrm{Ar}-\mathrm{CH}$, ring $\mathrm{A}), 7.95(\mathrm{~d}, 1 \mathrm{H}$, Ar-CH, ring A), 8.04 (d, $1 \mathrm{H}, \mathrm{Ar}-\mathrm{CH}$, ring A), 6.82 (s, 1H, Ar-CH, ring B), 6.76 (d, 1H, Ar-CH, ring B), 7.73 (d, 1H, Ar-CH, ring B), mass: $283.3(\mathrm{M}+1)$.

\section{2-phenyl-4H-chromen-4-one (2K)}

Molecular formula: $\mathrm{C}_{15} \mathrm{H}_{10} \mathrm{O}_{2}$, yield: $59 \%$, MP: $92-95^{\circ} \mathrm{C}$, IR $(\mathrm{KBr})$ $\mathrm{cm}^{-1}: 3045$ (-CH Aromatic str), 1645 (>C=0 str); ${ }^{1} \mathrm{H}$ NMR (DMSO-d6), $400 \mathrm{MHz}, \delta(\mathrm{ppm}): 7.07(\mathrm{~s}, 1 \mathrm{H}$, pyrone ring), $7.82(\mathrm{t}, 1 \mathrm{H}, \mathrm{Ar}-\mathrm{CH}$, ring $\mathrm{A})$, 7.50 (t, $1 \mathrm{H}, \mathrm{Ar}-\mathrm{CH}$, ring A), 8.12 (d, 1H, Ar-CH, ring A), 8.14 (d, 1H, Ar$\mathrm{CH}$, ring $\mathrm{A}), 7.85(\mathrm{~d}, 1 \mathrm{H}, \mathrm{Ar}-\mathrm{CH}$, ring $\mathrm{B}), 8.06(\mathrm{~d}, 1 \mathrm{H}, \mathrm{Ar}-\mathrm{CH}$, ring $\mathrm{B}), 7.63$, 7.61, $7.58(\mathrm{~m}, 3 \mathrm{H}, \mathrm{Ar}-\mathrm{CH}$, ring B), mass: $223.2(\mathrm{M}+1)$. 


\section{2-(3-bromophenyl)-4H-chromen-4-one (2L)}

Molecular formula: $\mathrm{C}_{15} \mathrm{H}_{9} \mathrm{BrO}_{2}$, yield: $68 \%$, MP: $112-115^{\circ} \mathrm{C}$, IR $(\mathrm{KBr})$ $\mathrm{cm}^{-1}: 3058$ (-CH Aromatic str), 1645 (>C=O str); ${ }^{1} \mathrm{H}$ NMR (DMSO-d6), $400 \mathrm{MHz}, \delta$ (ppm): 7.15 (s, 1H, pyrone ring), 7.57, 7.51 (m, 2H, Ar-CH, ring $\mathrm{A}), 7.50(\mathrm{t}, 1 \mathrm{H}, \mathrm{Ar}-\mathrm{CH}$, ring $\mathrm{A}), 7.85(\mathrm{~d}, 1 \mathrm{H}, \mathrm{Ar}-\mathrm{CH}$, ring $\mathrm{A}), 7.86(\mathrm{~d}$, $1 \mathrm{H}$, Ar- $\mathrm{CH}$, ring A), 8.32 (s, $1 \mathrm{H}$, Ar-CH, ring B), 8.15 (d, $1 \mathrm{H}, \mathrm{Ar}-\mathrm{CH}$, ring $\mathrm{B}$ ), $7.83\left(\mathrm{t}, 1 \mathrm{H}, \mathrm{Ar}-\mathrm{CH}\right.$, ring B), 8.05 (d, 1H, Ar-CH, ring B); mass: $301.2\left(\mathrm{M}^{+}\right)$.

\section{CONCLUSION}

The yield of chalcone derivatives (by green chemistry and conventional) were found to be in the range of $68-88 \%$ and by both the methods similar yield were obtained and the yield of flavone were lain between $58 \%$ and $85 \%$.

The purity of compounds was ascertained by melting point and TLC. The synthesized compounds were further established by IR, ${ }^{1} \mathrm{H}$ NMR, and mass spectral studies. Based on spectral data, it was proved that all synthesized chalcone and flavone derivatives meet the standard values of various spectral techniques.

\section{ACKNOWLEDGMENT}

The authors should thank the Gujarat Council on Science and Technology (GUJCOST, Gandhinagar, Gujarat, INDIA) for financial assistance (Under Minor Research Project).We are also acknowledged Ramanbhai Patel College of Pharmacy, Charotar University of Science and Technology, Changa (CHARUSAT) for providing the necessary infrastructure to carry out the research.

\section{REFERENCES}

1. Middleton E, Kandaswami C. The impact of plant flavonoids on mammalian biology: Implications for immunity, inflammation and cancer. In: Harborne JB, editor. The Flavonoids Advances in Research Since 1986. $1^{\text {st }}$ ed. London: Chapman and Hall; 1994. p. 619-52.

2. Harborne JB, Williams CA. Advances in flavonoid research since 1992. Phytochemistry 2000;55(6):481-504.

3. Pal D, Verma P. Flavonoids: A powerful and abundant source of antioxidants. Int J Pharm Pharm Sci 2013;5(3):95-8. 3. Available from: http://www.ijppsjournal.com/Vol5Issue3/7048.pdf.

4. Rice-Evans CA, Miller NJ, Paganga G. Structure-antioxidant activity relationships of flavonoids and phenolic acids. Free Radic Biol Med
1996;20(7):933-56.

5. Rice-Evans C. Flavonoid antioxidants. Curr Med Chem 2001;8(7):797-807.

6. Pietta PG. Flavonoids as antioxidants. J Nat Prod 2000;63(7):1035-42.

7. Chan EC, Pannangpetch P, Woodman OL. Relaxation to flavones and flavonols in rat isolated thoracic aorta: Mechanism of action and structureactivity relationships. J Cardiovasc Pharmacol 2000;35(2):326-33.

8. De Almeida ER, Xavier HS, Chaves TM, Couto GB, Aragao-Neto AC, Silva AR, et al. Anxiolytic and anticonvulsant effect of dioclenol flavonoids isolated from stem bark of Dioclea grandiflora on mice. Int J Appl Res Nat Prod 2009;2(4):44-51. Available from: http://www. ijarnp.org/index.php/ijarnp/article/view/40.

9. Liu YL, Ho DK, Cassady JM, Cook VM, Baird WM. Isolation of potential cancer chemopreventive agents from Eriodictyon californicum. J Nat Prod 1992;55(3):357-63.

10. Ramos S. Effects of dietary flavonoids on apoptotic pathways related to cancer chemoprevention. J Nutr Biochem 2007;18(7):427-42.

11. Ren W, Qiao Z, Wang H, Zhu L, Zhang L. Flavonoids: Promising anticancer agents. Med Res Rev 2003;23(4):519-34.

12. Shin JS, Kim KS, Kim MB, Jeong JH, Kim BK. Synthesis and hypoglycemic effect of chrysin derivatives. Bioorg Med Chem Lett 1999;9:869-74.

13. Dao TT, Chi YS, Kim J, Kim HP, Kim S, Park H. Synthesis and inhibitory activity against COX-2 catalyzed prostaglandin production of chrysin derivatives. Bioorg Med Chem Lett 2004;14(5):1165-7. 13. Available from: https://www.ncbi.nlm.nih.gov/pubmed/14980657.

14. Theja DN, Choudary TP, Reddy MI, Gupta A, Reddy KU. A facile synthesis of flavone derivatives used as potent anti-inflammatory agents. Int J Pharm Pharm Sci 2011;3(2):51-4.14.

15. Mostahar S, Alam S, Islam A. Cytotoxic and antimicrobial activities of some synthetic flavones. Indian J Chem 2006;45B:1478-86. Available from:15.http://www.nopr.niscair.res.in/handle/123456789/6487.

16. Tapas AR, Sakarkar DM, Kakde RB. Flavonoids as nutraceuticals: A review. Trop J Pharm Res 2008;7(3):1089-99. Available from: http:// www.bioline.org.br/pdf?pr08030.

17. Kakati D, Sarma JC. Microwave assisted solvent free synthesis of 1,3diphenylpropenones. Chem Cent J 2011;5(8):1-5. Available from: https://www.ccj.springeropen.com/articles/10.1186/1752-153X-5-8.

18. Sashidhara KV, Rosaiah JN, Kumar A. Iodine catalyse mild and efficient method for the synthesis of chalcones. Synth Commun 2009;39(3):2288-96. Available from: http:/www.tandfonline.com/doi/ abs/10.1080/00397910802654724?journalCode=lsyc20.

19. Li JT, Yang WZ, Wang SX, Li SH, Li SH. Improved synthesis of chalcones under ultrasound irradiation. Ultrason Sonochem 2002;9(5):237-9. 